\title{
ON BANACH SPACES OF VECTOR VALUED \\ CONTINUOUS FUNCTIONS
}

\section{Pilar Cembranos}

Let $K$ be a compact Hausdorff space and let $E$ be a Banach space. We denote by $C(K, E)$ the Banach space of all $E$-valued continuous functions defined on $K$, endowed with the supremum norm.

Recently, Talagrand [Israel J. Math. 44 (1983), 317-321] constructed a Banach space $E$ having the Dunford-Pettis property such that $C([0,1], E)$ fails to have the Dunford-Pettis property. So he answered negatively a question which was posed some years ago.

We prove in this paper that for a large class of compacts $K$ (the scattered compacts), $C(K, E)$ has either the Dunford-Pettis property, or the reciprocal Dunford-Pettis property, or the Dieudonné property, or property $V$ if and only if $E$ has the same property.

Also some properties of the operators defined on $C(K, E)$ are studied.

Received 23 May 1983. The author wishes to thank Professors F. Bombal and $\mathrm{J}$. Mendoza for their advice and encouragement.

Copyright Clearance Centre, Inc. Serial-fee code: 0004-9727/83 $\$ \mathrm{~A} 2.00+0.00$. 


\section{Introduction}

In 1953 Grothendieck [4] axiomatized some relevant properties of $C(K)$ spaces, introducing among others the so-called Dunford-Pettis, reciprocal Dunford-Pettis and Dieudonné properties. Later Pelczynski [7], in 1962, showed that $C(K)$ spaces enjoy another property, that he called property $V$, which can be defined on analogous terms to the preceding ones. Since then, these properties began to be studied on spaces of vector valued continuous functions, in particular on $C(K, E)$. The problem that was posed is the following: if $E$ has the Dunford-Pettis property, does $C(K, E)$ have this property too? The same question was asked for the other properties. This problem remained open for some years but, recently, Talagrand [9] constructed a Banach space $E$ having the Dunford-Pettis property such that $C([0,1], E)$ fails to have the Dunford-Pettis property. Talagrand's work shows the interest of looking for conditions on spaces $K$ and $E$ to obtain an affirmative answer to the posed problem.

We prove in this paper that for a large class of compacts $K$ (the scattered compacts), it is enough to take a Banach space $E$ having one of the mentioned properties to insure that $C(K, E)$ has the same property.

Since the study of such properties on a space is closely related to the study of the operators defined on it, we devote the first part of our work to show some properties of the operators defined on $C(K, E)$.

Throughout the paper $E$ and $F$ are Banach spaces, $K$ is a compact Hausdorff space and $\Sigma$ is the $\sigma$-field of Borel subsets of $K . C(K, E)$ is the Banach space of all E-valued continuous functions on $K$, and $B(\Sigma, E)$ is the Banach space of all functions $\varphi: K \rightarrow E$ which are the uniform limit of a sequence of $\Sigma$-simple functions. Both spaces are endowed with the supremum norm. The term "operator" means a bounded linear operator. We denote by $L(E, F)$ the space of all operators from $E$ to $F$.

It is well known that an operator $T: C(K, E) \rightarrow F$ may be represented as an integral with respect to a finitely additive set function $m: \Sigma \rightarrow L\left(E, F^{\prime \prime}\right)$ having finite semivariation on $K \quad(\hat{m}(K)<+\infty)$ and so that $\|T\|=\hat{m}(K)$ (see, for example, [2], p. 182); $m$ is called the representing measure of $T$.

A compact space $K$ is scattered if every subset $A$ of $K$ has a 
point relatively isolated in $A$. The class of compact scattered spaces includes all countable compact spaces and all compact ordinals (with the interval topology).

2. Some properties of the operators defined on $C(K, E)$

An operator $T: C(K, E) \rightarrow F$ whose representing measure $m$ has its values in $L(E, F)$ determines an extension $\hat{T}: B(\Sigma, E) \rightarrow F$ given by

$$
\hat{T}(\varphi)=\int_{K} \varphi d m, \varphi \in B(\Sigma, E),
$$

with $\|\hat{T}\|=\|T\| \quad($ see $[1]$, Theorem 2).

Batt and Berg [1] showed that an operator $T: C(K, E) \rightarrow F$ is weakly compact if and only if its extension $\hat{T}$ to $B(\Sigma, E)$ is weakly compact.

We shall prove that one can obtain analogous results for other properties of $T$ when $K$ is metrizable.

THEOREM 1. Let $K$ be metrizable. Then an operator $T: C(K, E) \rightarrow E$ is unconditionally converging if and only if its extension $\hat{T}$ to $B(\Sigma, E)$ is unconditionally converging.

Proof. Let $T: C(K, E) \rightarrow F$ be an unconditionally converging operator. Then, by Theorem 3 and Lemma 2 of [3], its representing measure $m$ has its values in $L(E, F)$ and there is a finite non negative measure $\lambda$ on $\Sigma$ so that

$$
\lim _{\lambda(A) \rightarrow 0} \hat{m}(A)=0
$$

If we suppose that $\hat{T}: B(\Sigma, E) \rightarrow F$ is not unconditionally converging, then there exist $\varepsilon>0$ and a weakly unconditionally convergent series $\sum \varphi_{n}$ in $B(\Sigma, E)$ such that

$$
\left\|\hat{T}\left(\varphi_{n}\right)\right\|>\varepsilon \text { for all } n \in \mathbb{N}
$$

It is well known that a series $\sum x_{n}$ in a Banach space is weakly unconditionaly convergent if and only if the set

$$
\left\{\sum_{n \in \sigma} x_{n}: \sigma \subset \mathbb{N} \text { is finite }\right\}
$$


is bounded. Therefore there is $M>0$ verifying

$$
\left\|\sum_{n \in \sigma} \varphi_{n}\right\|<M \text { for all finite subsets } \sigma \text { of } N \text {. }
$$

By (1) we can choose $\delta>0, \delta<\lambda(K)$, such that

$$
\hat{m}(A)<\frac{\varepsilon}{4 M} \text { for } A \in \Sigma \text { with } \lambda(A)<\delta \text {. }
$$

According to Lusin's theorem, for each $n \in \mathbb{N}$, there exists a compact $K_{n} \subset K$ such that $\lambda\left(K \backslash K_{n}\right)<\delta / 2^{n}$ and $\left.\varphi_{n}\right|_{K_{n}}$ (the restriction of $\varphi_{n}$ to $\left.K_{n}\right)$ is continuous. Put $K_{0}=\prod_{n=1}^{\infty} K_{n}$. Then $\lambda\left(K \backslash K_{0}\right)<\delta$, and $K_{0} \neq \emptyset$ because $\delta<\lambda(K)$. Let us denote $\Phi_{n}=\left.\varphi_{n}\right|_{K_{0}}$ for $n \in \mathbf{N}$. By (3) the series $\sum \Phi_{n}$ is weakly unconditionally convergent in $C\left(K_{0}, E\right)$.

Now, by the Borsuk-Dungundji theorem (see 21.1 .4 of [8]), there is an operator $S: C\left(K_{0}, E\right) \rightarrow C(K, E)$, with $\|S\|=1$, so that $\left.S(\Phi)\right|_{K_{0}}=\Phi$ for every $\Phi \in C\left(K_{0}, E\right)$. The operator $T S: C\left(K_{0}, E\right) \rightarrow F$ is unconditionally converging. However, the series $\sum T S\left(\Phi_{n}\right)$ does not converge in $F$ because, by (2), (3) and (4), for each $n \in \mathbb{N}$,

$$
\begin{aligned}
\left\|T S\left(\Phi_{n}\right)\right\| & =\left\|\int_{K} S\left(\Phi_{n}\right) d m\right\| \geq\left\|\int_{K_{0}} \varphi_{n} d m\right\|-\left\|\int_{K \backslash K_{0}} S\left(\Phi_{n}\right) d m\right\| \\
& \geq\left\|\int_{K} \Phi_{n} d m\right\|-\left\|\int_{K \backslash K_{0}} \varphi_{n} d m\right\|-\left\|S\left(\Phi_{n}\right)\right\| \hat{m}\left(K \backslash K_{0}\right) \\
& \geq\left\|\hat{T}\left(\varphi_{n}\right)\right\|-2 \hat{m m}\left(K \backslash K_{0}\right)>\varepsilon-\frac{\varepsilon}{2}=\frac{\varepsilon}{2} .
\end{aligned}
$$

This contradiction shows that if $T: C(K, E) \rightarrow F$ is unconditionally converging then $\hat{T}$ is also. The converse is obvious.

THEOREM 2. Let $K$ be metrizable. Then an operator $T: C(K, E) \rightarrow F$ transforms weakly Cauchy sequences into weakly convergent ones if and only if its extension $\hat{T}$ to $B(\Sigma, E)$ transforms weakly Cauchy sequences into weakly convergent ones. 
Proof. Let $T: C(K, E) \rightarrow F$ be an operator which maps weakly Cauchy sequences into weakly convergent sequences. Then $T$ is unconditionally converging. Let $m$ and $\lambda$ be as in the preceding proof. Let $\left(\varphi_{n}\right)$ be a weakly Cauchy sequence in $B(\Sigma, E)$ and let $y^{\prime \prime} \in F^{\prime \prime}$ so that $\left(\hat{T}\left(\varphi_{n}\right)\right)$ is $\sigma\left(E^{\prime \prime}, F^{\prime}\right)$-convergent to $y^{\prime \prime}$. If we suppose that $\left(\hat{T}\left(\varphi_{n}\right)\right)$ is not weakly convergent in $F$ then $y^{\prime \prime} \mid F$. By using Grothendieck's completeness theorem (see 3.11 .4 of [5]) it follows that there exist $\varepsilon>0$ and a net $\left(y_{i}^{\prime}\right)_{i \in I}$ in the unit ball of $F^{\prime}$ which is $\sigma\left(F^{\prime}, F\right)$-convergent to zero such that

$$
\left|\left\langle y_{i}^{\prime}, y^{\prime \prime}\right\rangle\right|>\varepsilon \text { for all } i \in I
$$

Choose $\delta>0, \delta<\lambda(K)$, verifying

$$
\hat{m}(A)<\frac{\varepsilon}{8 \mathrm{sup}\left\|\varphi_{n}\right\|} \text { for } A \in \Sigma \text { with } \lambda(A)<\delta .
$$

Similarly as in the preceding proof we can take a non empty compact $K_{0} \subset K$ so that $\lambda\left(K \backslash K_{0}\right)<\delta$ and $\Phi_{n}=\left.\varphi_{n}\right|_{K_{0}}$ is continuous for $n \in \mathbb{N}$, and an operator $S: C\left(K_{0}, E\right) \rightarrow C(K, E)$, with $\|S\|=1$, such that $\left.S(\Phi)\right|_{K_{0}}=\Phi$ for $\Phi \in C\left(K_{0}, E\right)$. For each $t \in K_{0}$ the sequence $\left(\Phi_{n}(t)\right)$ is weakly Cauchy in $E$, therefore, according to Theorem 9 of [3], $\left(\Phi_{n}\right)$ is weakly Cauchy in $C\left(K_{0}, E\right)$. Then $\left(T S\left(\Phi_{n}\right)\right)$ is weakly convergent to an element $y \in F$. Since $\left(y_{i}^{\prime}\right)_{i \in I}$ is $\sigma\left(F^{\prime}, F\right)$-convergent to zero there exists $i_{0} \in I$ so that

$$
\left|\left\langle y, y_{i}^{\prime}\right\rangle\right|<\varepsilon / 6 \text { for all } i \geq i_{0} \text {. }
$$

Let $i \geq i_{0} ;$ then there is $n \in \mathbb{N}$ verifying

$$
\left|\left\langle\hat{T}\left(\varphi_{n}\right)-y^{\prime \prime}, y_{i}^{\prime}\right\rangle\right|<\varepsilon / 6 \text { and }\left|\left\langle T S\left(\Phi_{n}\right)-y, y_{i}^{\prime}\right\rangle\right|<\varepsilon / 6 .
$$

Thus we have 


$$
\begin{aligned}
\left|\left\langle y^{\prime \prime}, y_{i}^{\prime}\right\rangle\right| & \leq\left|\left\langle y^{\prime \prime}-\hat{T}\left(\varphi_{n}\right), y_{i}^{\prime}\right\rangle\right|+\left|\left\langle\hat{T}\left(\varphi_{n}\right)-T S\left(\Phi_{n}\right), y_{i}^{\prime}\right\rangle\right| \\
& \quad+\left|\left\langle T S\left(\Phi_{n}\right)-y, y_{i}^{\prime}\right\rangle\right|+\left|\left\langle y, y_{i}^{\prime}\right\rangle\right| \\
& \leq \frac{\varepsilon}{2}+\left\|y_{i}^{\prime}\right\|\left\|\hat{T}\left(\varphi_{n}\right)-T S\left(\Phi_{n}\right)\right\| \\
& \leq \frac{\varepsilon}{2}+2\left\|K_{0} \mid \varphi_{n}\right\| \hat{m}\left(K \backslash K_{0}\right)<\frac{3}{4} \varepsilon .
\end{aligned}
$$

But this contradicts (5).

The converse is clear.

THEOREM 3. Let $K$ be metrizable. Then an operator $T: C(K, E) \rightarrow F$ maps weakly convergent sequences into norm convergent sequences if and only if its extension $\hat{T}$ to $B(\Sigma, E)$ maps weakly convergent sequences into norm convergent ones.

Proof. Let $T: C(K, E) \rightarrow F$ be an operator which maps weakly convergent sequences into norm convergent ones. Then $T$ is unconditionally converging. Let $m$ and $\lambda$ be as in the proof of Theorem 1. Let $\left(\varphi_{n}\right)$ be a sequence in $B(\Sigma, E)$ which is weakly convergent to zero. Suppose that there exist $\varepsilon>0$ and a subsequence of $\left(\varphi_{n}\right)$ (which we still denote by $\left(\varphi_{n}\right)$ ) so that

$$
\left\|\hat{T}\left(\varphi_{n}\right)\right\|>\varepsilon \text { for all } n \in \mathbb{N} \text {. }
$$

Choose $\delta>0, \delta<\lambda(K)$, verifying

$$
\hat{m}(A)<\frac{\varepsilon}{\operatorname{ssup}\left\|\varphi_{n}\right\|} \text { for } A \in \Sigma \text { with } \lambda(A)<\delta .
$$

Reasoning as in the proof of Theorem 1 , there exist a non empty compact $K_{0} \subset K$, with $\lambda\left(K \backslash K_{0}\right)<\delta$, such that $\Phi_{n}=\left.\varphi_{n}\right|_{K_{0}}$ is continuous for all $n \in \mathbb{N}$, and an operator $S: C\left(K_{0}, E\right) \rightarrow C(K, E)$, with $\|S\|=1$, so that $\left.S(\Phi)\right|_{K_{0}}=\Phi$ for $\Phi \in C\left(K_{0}, E\right)$. According to Theorem 9 of [3], $\left(\Phi_{n}\right)$ is weakly convergent to zero in $C\left(K_{0}, E\right)$. Then $\left(T S\left(\Phi_{n}\right)\right)$ is norm convergent to zero and there exists $n_{0} \in \mathbb{N}$ such that 


$$
\left\|T S\left(\Phi_{n}\right)\right\|<\varepsilon / 3 \text { for all } n \geq n_{0} \text {. }
$$

Thus if $n \geq n_{0}$ one has

$$
\begin{aligned}
\left\|\hat{T}\left(\varphi_{n}\right)\right\| & \leq\left\|\hat{T}\left(\varphi_{n}\right)-T S\left(\Phi_{n}\right)\right\|+\left\|T S\left(\Phi_{n}\right)\right\| \\
& <\left\|\int_{K \backslash K_{0}}\left(\varphi_{n}-S\left(\Phi_{n}\right)\right) d m\right\|+\frac{\varepsilon}{3} \\
& \leq 2\left\|\varphi_{n}\right\| \hat{m}\left(K \backslash K_{0}\right)+\frac{\varepsilon}{3}<\frac{2}{3} \varepsilon .
\end{aligned}
$$

But this contradicts (6).

The converse is obvious.

\section{Some properties on $C(K, E)$}

THEOREM 4. If $K$ is scattered then $C(K, E)$ has the Dunford-Pettis property if and only if $E$ has.

Proof. It is clear that if $C(K, E)$ has the Dunford-Pettis property then $E$ has it too.

Suppose that $E$ has the Dunford-Pettis property.

(A) If $K$ is metrizable then, by 8.5 .5 of [8], $K$ is countable. Now the proof of Theorem 13 (a) of [3] works the same here.

(B) For a general $K$, let $T: C(K, E) \rightarrow F$ be a weakly compact operator and let $\left(\Phi_{n}\right)$ be a sequence in $C(K, E)$ weakly convergent to zero. Similarly as in [1], page 236, we can construct a metrizable quotient space $\bar{K}$ of $K$ and a sequence $\left(\Phi_{n}\right) \subset C(\bar{K}, E)$ such that $\Phi_{n}(\pi(t))=\Phi_{n}(t)$ for all $t \in K$ and $n \in \mathbb{N}$, where $\pi: K \rightarrow \bar{K}$ is the canonical mapping. By 8.5 .3 of [8], $\bar{K}$ is scattered, and Theorem 9 of [3] implies that $\left(\bar{\Phi}_{n}\right)$ is weakly convergent to zero in $C(\bar{K}, E)$. If we consider the operator $\bar{T}: C(\bar{K}, E) \rightarrow F$ defined by $\bar{T}(\bar{\Phi})=T(\bar{\Phi} \cdot \pi)$ for $\bar{\Phi} \in C(\bar{K}, E)$, it follows from $(\mathrm{A})$ that $\lim \left\|\bar{T}_{n}\left(\bar{\Phi}_{n}\right)\right\|=0$. Since $T\left(\Phi_{n}\right)=\bar{T}\left(\bar{\Phi}_{n}\right)$ for all $n \in \mathbb{N}$, we conclude that $C(K, E)$ has the DunfordPettis property.

Note that if $C(K, E)$ has the Dunford-Pettis property when $K$ is 
metrizable then, as in (B) of the preceding proof, it follows that $C(K, E)$ has the Dunford-Pettis property for every compact $K$. Therefore an immediate consequence of $8.5 .7,21.5 .10$ and 21.5 .1 of [8], and Theorem 4 is the following:

COROLLARY 5. $C(K, E)$ has the Dhonford-Pettis property for every compact $K$ if and only if $C([0,1], E)$ has the Dronford-Pettis property.

Recall that if $m$ is the representing measure of an operator $T: C(K, E) \rightarrow F$, it is said that the semivariation $\hat{m}$ of $m$ is continuous on $\Sigma$ if for every decreasing sequence $\left(A_{n}\right)$ in $\Sigma$, with $\bigcap_{n} A_{n}=\emptyset$, there is $\underset{n}{\lim } \hat{m}\left(A_{n}\right)=0$.

LEMMA 6. Let $K$ be a metrizable scattered compact space and let $T: C(K, E) \rightarrow F$ be an operator whose representing measure $m$ verifies

(i) $m(\Sigma) \subset L(E, E)$,

(ii) $m(A): E \rightarrow F$ is weakly compact for each $A \in \Sigma$,

(iii) $\hat{m}$ is continuous on $\Sigma$.

Then $T$ is weakly compact.

Proof. By 8.5 .5 of $[8], K$ is countable. Put $K=\left\{t_{i}: i \in \mathbb{N}\right\}$. Let $\left(\Phi_{n}\right)$ be a bounded sequence in $C(K, E)$. For each $n \in \mathbb{N}$ we can take a $\Sigma$-simple function $\varphi_{n} \in B(\Sigma, E)$ so that $\left\|\varphi_{n}-\Phi_{n}\right\|<1 / n$. According to condition ( $i i)$, for every $i \in \mathbb{N}$ the sequence $\left(m\left(\left\{t_{i}\right\}\right)\left(\varphi_{n}\left(t_{i}\right\}\right)\right)_{n}$ has a weakly convergent subsequence. This fact enables us to use Cantor's diagonal argument to extract a subsequence of $\left(\varphi_{n}\right)$ (which we still denote by $\left.\left(\varphi_{n}\right)\right)$ such that $\left(m\left(\left\{t_{i}\right\}\right)\left(\varphi_{n}\left(t_{i}\right\}\right)\right)_{n}$ is weakly convergent in $F$ for all $i \in \mathbb{N}$. For each $i \in \mathbb{N}$ let $y_{i}$ be the $\sigma\left(E, F^{\prime}\right)$-limit of $\left(m\left(\left\{t_{i}\right\}\right)\left(\varphi_{n}\left(t_{i}\right)\right)\right)_{n}$. The series $\sum y_{i}$ converges in $F$. To prove this suppose that there exist $\varepsilon>0$ and a sequence $\left(\sigma_{j}\right)$ of finite subscts of $N$, wilh $\max \sigma_{j}<\min \sigma_{j+1}$ for $j \in N$, such that

$$
\left\|\sum_{i \in \sigma_{j}} y_{i}\right\|>\varepsilon \text { for all } j \in N \text {. }
$$


Hence for every $j \in \mathbf{N}$ we can choose $y_{j}^{\prime}$ in the unit ball of $F^{\prime}$ verifying

$$
\left|\left\{\sum_{i \in \sigma_{j}} y_{i}, y_{j}^{\prime}\right\rangle\right|>\varepsilon
$$

Thus it follows from the choice of $\left(y_{i}\right)$ that there is an increasing sequence $\left(n_{j}\right) \subset N$ such that

$$
\left|\left\langle\sum_{i \in \sigma_{j}} m\left(\left\{t_{i}\right\}\right)\left(\varphi_{n_{j}}\left(t_{i}\right)\right), y_{j}^{\prime}\right\rangle\right|>\varepsilon \text { for } j \in \mathbb{N} .
$$

We set $A_{j}=\bigcup_{k=j}^{\infty}\left\{t_{i}: i \in \sigma_{k}\right\}, j \in \mathbb{N}$. Then one has

$$
\begin{aligned}
\hat{m}\left(A_{j}\right) & \geq \hat{m}\left(\left\{t_{i}: i \in \sigma_{j}\right\}\right) \\
& \geq \frac{1}{\sup \left\|\varphi_{n}\right\|}\left\|\sum_{i \in \sigma_{j}} m\left(\left\{t_{i}\right\}\right)\left(\varphi_{n_{j}}\left(t_{i}\right)\right)\right\| \\
& >\frac{\varepsilon}{\sup \left\|\varphi_{n}\right\|} .
\end{aligned}
$$

This contradicts condition (iii) since $\left(A_{j}\right)$ is a decreasing sequence in $\sum$ with $\bigcap_{j} A_{j}=\varnothing$. Therefore $\sum y_{i}$ converges in $F$. Let $y=\sum y_{i}$. We claim that $\left(T\left(\Phi_{n}\right)\right)$ is weakly convergent to $y$. Let $\varepsilon>0$ and let $y^{\prime} \in F^{\prime}$ with $\left\|y^{\prime}\right\| \leq I$, then there exist $n_{0} \in \mathbb{N}$ and $k \in \mathbb{N}$ such that

$$
\left\|_{i>n_{0}} y_{i}\right\|<\varepsilon / 4, \hat{m}\left(\left\{t_{i}: i>n_{0}\right\}\right)<\frac{\varepsilon}{4 \sup \left\|\varphi_{n}\right\|+1}, \frac{1}{k}<\frac{\varepsilon}{4\|T\|+1} \text {, }
$$

and

$$
\left|\left\langle\sum_{i=1}^{n_{0}} m\left(\left\{t_{i}\right\}\right)\left(\varphi_{n}\left(t_{i}\right)\right)-\sum_{i=1}^{n_{0}} y_{i}, y^{\prime}\right\rangle\right|<\varepsilon / 4 \text { for all } n \geq k .
$$

If $\hat{T}$ is the extension of $T$ to $B(\Sigma, E)$ and we put $B=\left\{t_{i}: i>n_{0}\right\}$ then for each $n \geq k$ one has 


$$
\begin{aligned}
\left|\left\langle T\left(\Phi_{n}\right)-y, y^{\prime}\right\rangle\right| & \leq\left|\left\langle\hat{T}\left(\Phi_{n}-\varphi_{n}\right), y^{\prime}\right\rangle\right|+\left|\left\langle\hat{T}\left(\varphi_{n}\right)-y, y^{\prime}\right\rangle\right| \\
& \left.<\frac{\varepsilon}{4}+\left|\left\langle\int_{B} \varphi_{n} d m, y^{\prime}\right\rangle\right|+\left|\langle| \int_{K \backslash B} \varphi_{n} d m\right)-y, y^{\prime}\right\rangle \mid \\
& \leq \frac{\varepsilon}{4}+\left\|\varphi_{n}\right\| \hat{m}(B)+\left|\left\langle\sum_{i>n_{0}} y_{i}, y^{\prime}\right\rangle\right| \\
& +\left|\left\langle\sum_{i=1}^{n_{0}} m\left(\left\{t_{i}\right\}\right)\left(\varphi_{n}\left(t_{i}\right)\right)-\sum_{i=1}^{n_{0}} y_{i}, y^{\prime}\right\rangle\right|<\varepsilon .
\end{aligned}
$$

Thus we conclude that $T$ is weakly compact.

REMARK. Note that conditions (i), (ii) and (iii) of Lemma 6 are necessary for an operator $T: C(K, E) \rightarrow F$ to be weakly compact but, in general, they are not sufficient.

THEOREM 7. Suppose that $K$ is scattered. Then $C(K, E)$ has either the reciprocal Dunford-Pettis property, or the Dieudonne property, or property $V$ if and only if $E$ has the same property.

Proof. We only consider the case of the Dieudonné property. The rest can be proved in the same way.

If $C(K, E)$ has the Dieudonné property it is clear that $E$ has it too.

Assume that $E$ has the Dieudonne property.

(A) Let us first suppose that $K$ is metrizable. Let $T: C(K, E) \rightarrow F$ be an operator which maps weakly Cauchy sequences into weakly convergent ones. Then $T$ is unconditionally converging and, by Theorem 3 of [3], its representing measure $m$ verifies conditions ( $i$ ) and (iii) of Lemma 6. For each $A \in \Sigma$ the map $\tau_{A}: E \rightarrow B(\Sigma, E)$ defined by $\tau_{A}(x)=x \chi_{A}$ is a bounded linear map. So it follows from Theorem 2 that the operator $m(A)=\hat{T}_{A}: E \rightarrow F$ transforms weakly Cauchy sequences into weakly convergent sequences. Since $E$ has the Dieudonné property $m(A): E \rightarrow F$ is weakly compact. Therefore, according to Lemma $6, T$ is weakly compact.

(B) For a general $K$ the same method used in [1], page 236, and the fact that a metrizable quotient space of a scattered space is scattered (see 8.5 .3 of $[8]$ ), proves that $C(K, E)$ has the Dieudonné property. 
The next result is an immediate consequence of $8.5 .7,21.5 .1$ and 21.5 .10 of [8] and Theorem 7 , by means of the standard reduction to the case $K$ metrizable.

COROLLARY 8. $C(K, E)$ has either the reciprocal Dronford-Pettis property, or the Dieudonne property, or property $V$ for every compact $K$ if and only if $C([0,1], E)$ has the same property.

\section{References}

[1] Jürgen Batt and E. Jeffrey Berg, "Linear bounded transformations on the space of continuous functions", J. Funct. Anaz. 4 (1969), 215-239.

[2] J. Diestel and J.J. UhI, Jr., Vector measures (Mathematical Surveys, 15. American Mathematical Society, Providence, Rhode Island, 1977).

[3] I van Dobrakov, "On representation of linear operators on $C_{0}(T, X)$ ", Caechoslovak Math. J. 21 (96) (1971), 13-30.

[4] A. Grothendieck, "Sur les applications lineaires faiblement compactes d'espaces du type $C(K)$ ", Canad. J. Math. 5 (1953), 129-173.

[5] John Horváth, Topological vector spaces and distributions, Volume I (Addison-Wesley, Reading, Massachusetts; Palo Alto; London; 1966).

[6] Joram Lindenstrauss, Lior Tzafriri, Classical Banach spaces. I. Sequence spaces (Ergebnisse der Mathematik und ihrer Grenzgebiete, 92. Springer-Verlag, Berlin, Heidelberg, New York, 1977.

[7] A. Pelczyński, "Banach spaces on which every unconditionally converging operator is weakly compact", Bull. Acad. Polon. Sci. Ser. Sci. Math. Astronom. Phys. 10 (1962), 641-648.

[8] Zbigniew Semadeni, Banach spaces of continuous functions (Monografie Matematyczne, 55. PWN - Polish Scientific Publishers, Warszawa, 1971). 
[9] M. Talagrand, "La propriété de Dunford-Pettis dans $C(K, E)$ et $L^{1}(E) "$, Israel J. Math. 44 (1983), 317-321.

Departamento de Teoria de Funciones, Facultad de Ciencias Matematicas, Universidad Complutense de Madrid, Madrid 3,

Spain. 